\title{
Fine Structure in Magnetization of Individual Fluxoid States
}

\author{
A. K. Geim, ${ }^{1}$ S. V. Dubonos, ${ }^{2,1}$ J. J. Palacios, ${ }^{3}$ I. V. Grigorieva, ${ }^{1}$ M. Henini,${ }^{4}$ and J. J. Schermer ${ }^{1}$ \\ ${ }^{1}$ University of Nijmegen, Toernooiveld 1, 6525 ED Nijmegen, The Netherlands \\ ${ }^{2}$ Institute for Microelectronics Technology, 142432 Chernogolovka, Russia \\ ${ }^{3}$ Departamento de Física Teórica de la Materia Condensada, Universidad Autónoma de Madrid, \\ Cantoblanco, Madrid 28049, Spain \\ ${ }^{4}$ School of Physics, University of Nottingham, Nottingham NG7 2RD, United Kingdom
}

(Received 15 December 1999)

\begin{abstract}
Each time a vortex enters or exits a small superconductor, a different fluxoid state develops which can be characterized by its vorticity, i.e., the number of fluxoids inside. We have studied magnetization response of such individual states and found clear signatures of first and second order transitions within the states, which reveal the existence of distinct vortex phases for a fixed number of fluxoids. We attribute the transitions to the merger of individual vortices into a single giant vortex and switching between different arrays of vortices.
\end{abstract}

PACS numbers: 74.25.Dw, 74.60.Ge, 74.76.Db

Mesoscopic superconductors of the size comparable to the superconducting coherence length are able to accommodate only a small number of vortices before their superconductivity is destroyed and, accordingly, they are referred to as few-fluxoid superconductors (FFS) [1-4]. In comparison to all the progress made on other mesoscopic systems of similar (submicron) sizes, surprisingly little remains known about FFS. Until recently, experimental studies on this system were essentially limited to its behavior at the normal-superconducting phase boundary [5-8]. The ballistic Hall magnetometry [9] has made it possible to investigate individual FFS inside the superconducting state, away from the phase boundary. Using this technique, it has been shown [1-4] that a mesoscopic superconductor does not exhibit the standard magnetization dependences but follows a rather dissimilar set of curves so that each fluxoid state exhibits its own magnetization dependence.

Here we report a new feature of the mesoscopic superconductivity: The existence of different vortex phases within individual fluxoid states. The phases reveal themselves via branching and kinks on magnetization curves for a given vorticity. The kinks indicate second-order transitions, in which several Abrikosov vortices coalesce into a single giant vortex. Such phase transitions are being intensively discussed in theory [3,4,10-12], but this is their first experimental observation. The unexpected but more pronounced first-order transitions associated with the branching of the curves are attributed to switching between different arrays of the same number of vortices.

For the purpose of this report, we discuss the behavior of $\mathrm{Al}$ disks with diameters $d$ less than $4 \mu \mathrm{m}$ and a thickness of about $0.1 \mu \mathrm{m}$, which, in our experience, present an optimum system for studies of FFS. Such disks (a) can accommodate only a few dozen vortices so that all their fluxoid states can be accurately measured within reasonable time; (b) exhibit a rather strong magnetization response due to a short penetration length $\lambda$; and (c) show no discernible bulk pinning. The disks' magnetization was measured by ballistic Hall magnetometry [9]. For brevity, one can simply consider our Hall magnetometer as a fluxmeter with a square detection loop of size $w$, in the center of which a superconducting disk is placed. We present our measurements in terms of the (area) magnetization $4 \pi M=\langle B\rangle-H$, where $\langle B\rangle$ is the average magnetic field within the central area $(w \times w)$, which is directly measured in the experiment, and $H$ is the applied field. The absolute value of $M$ depends on the filling factor $d / w$ (Ref. [9]). In comparison to the previous work [1], the sensitivity is significantly improved so that not only individual fluxoid curves, but also fine features on them (previously concealed by noise) are resolved [Fig. 1(a)].

Two examples of magnetization response for such mesoscopic disks are shown in Fig. 1. The disks have $T_{\mathrm{C}} \approx$ $1.2 \mathrm{~K}$ and the third (surface) critical field extrapolated to zero temperature, $H_{\mathrm{c} 3}(0)$, of $\approx 180 \mathrm{G}$, as found in magnetization measurements. Alternatively, measuring the resistivity of a macroscopic film evaporated simultaneously with the disks, we find the bulk critical field $H_{\mathrm{c} 2}(0) \approx$ $105 \mathrm{G}$. The coherence length $\xi(0)$ is estimated to be $\approx 0.25 \mu \mathrm{m}$ and $\lambda(0) \approx 70 \mathrm{~nm}$ [1], i.e., the material is a type-I superconductor $(\kappa=\lambda / \xi \approx 0.3)$. One has to bear in mind, however, that thin films in a perpendicular magnetic field behave more like type-II superconductors and are expected to exhibit vortex structures $[10,13]$. We intentionally work with films having $\kappa$ as low as possible. In fact, we can move into the true type-II regime by using less pure $\mathrm{Al}$, but our tentative measurements showed no unexpected changes in overall behavior, at least, up to $\kappa \approx 1$. At the same time, the reduced screening due to unavoidably larger $\lambda$ for larger $\kappa$ leads to rapid deterioration of the experimental resolution.

Figure 1(a) shows a series of well-resolved magnetization curves. Each curve can be described by a fluxoid number $L$ (vorticity) that determines how many times the phase changes by $2 \pi$ along the sample's circumference 

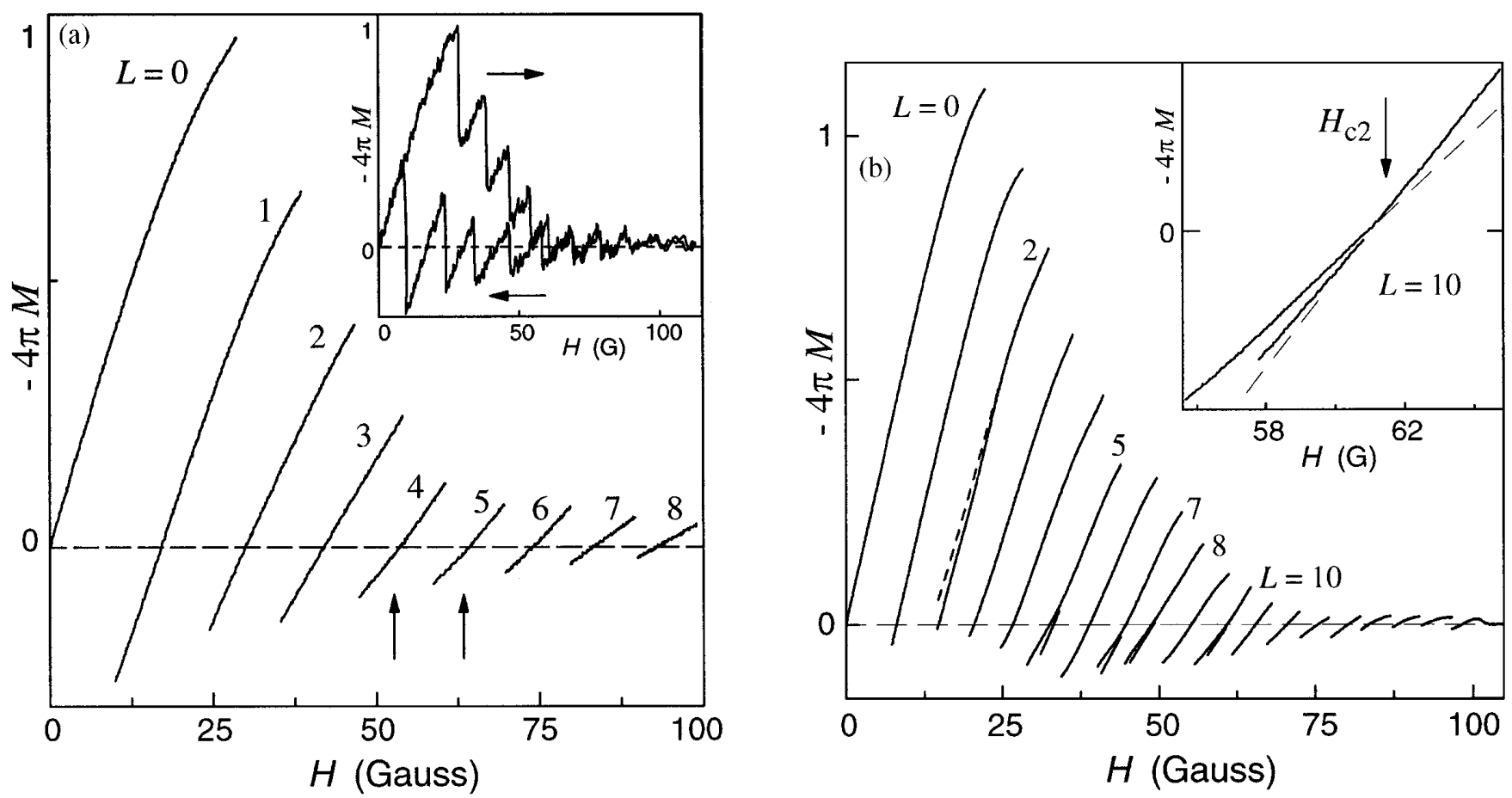

FIG. 1. Magnetizations of Al disks of diameters 1.5 (a) and $2.4 \mu \mathrm{m}$ (b) in magnetic field along the disk axis at $T \approx 0.5 \mathrm{~K}$. The magnetometer width is $\approx 2.5 \mu \mathrm{m}$. The inset in panel (a) shows the magnetization response when the field is swept up continuously from zero to high fields and then back. Panel (a) also allows comparison between the resolution previously achieved (inset) and the present one (main figure). The improvement is mostly due to the use of a few seconds of infrared illumination of the Hall probes from their back through the GaAs substrate. The inset in panel (b) magnifies one of the curves exhibiting branching and a kink, which correspond to two different arrays of ten vortices and their merger in a giant vortex. The dashed curves in the inset indicate the change of the slope.

[1-4]. When the magnetic field is swept continuously, the magnetization evolves along one of the fluxoid curves until it reaches its end and jumps to the next curve, belonging to another fluxoid state. Then, the process repeats itself all over again. An important feature of FFS is the existence of several fluxoid states for the same $H$. Apparently, only one of such states is thermodynamically stable. The ground state comprises nearly the whole low-field curve ( $L=0$; the Meissner state), and smaller segments of the other curves close to their upper (diamagnetic) ends [2-4]. Other states in the multiple-choice situations are metastable but can persist for many hours and days. This metastability is inherent [1-4] and leads to the hysteresis for continuous field sweeps. On the other hand, each of the fluxoid curves is completely reproducible (no hysteresis), indicating the virtual absence of pinning.

The majority of fluxoid curves in Fig. 1 are bent at the diamagnetic end, which is most clearly seen for the Meissner state $(L=0)$. In addition to the smooth curvature, there are a number of fine details that we are able to distinguish due to the improved resolution. A first feature to notice is additional curves that look like splitting or branching for $L=2,5,7,8$, and 10 . As only integer fluxoid numbers are allowed, we refer to the additional fragments as substates of the corresponding states. No branching has been observed for disks with the maximum allowed $L$ less than 10 [cf. Fig. 1(a)]. With increasing tem- perature, the extra segments rapidly become shorter and disappear above $0.8 \mathrm{~K}$. The substates at $L=5,7,8$, and 10 in Fig. 1 persist for many hours but could only be reached on rare occasions by jumping from the corresponding $(L+1)$-states during down sweeps. If the field is swept beyond the stability range of substates, they usually switch to the main state that they belong to. This always happens with a jump in magnetization (first-order transition) and, accordingly, the substate curves do not touch the main curves. Furthermore, the substate curves are not a simple continuation of the main curves but have somewhat shallower slopes [Fig. 1(b)].

Another new feature we observed is kinks on the curves which can already be discerned in Fig. 1(a) (vertical arrows). For a better view, Figs. 1(b) and 2(a) magnify two of such kinks for the larger disk. The $L=7$ state always exhibits the most pronounced kink where the slope changes by $\approx 40 \%$. Weaker kinks have been observed for many other fluxoid states (namely, $L=2,4,5,8,9,10,11,13$, and 17), and we cannot exclude the presence of other unresolved kinks, e.g., at $L=3$ and 6 . However, neither of our samples showed any evidence for kinks or branching for $L=0$ or 1 and for all $L$ above $H_{\mathrm{c} 2}$.

The substate at $L=2$ turns out to be a special case. It is found at the low-field side of the main curve, in contrast to the location of the other substates. This substate is rather unstable and falls on the main curve within several minutes 

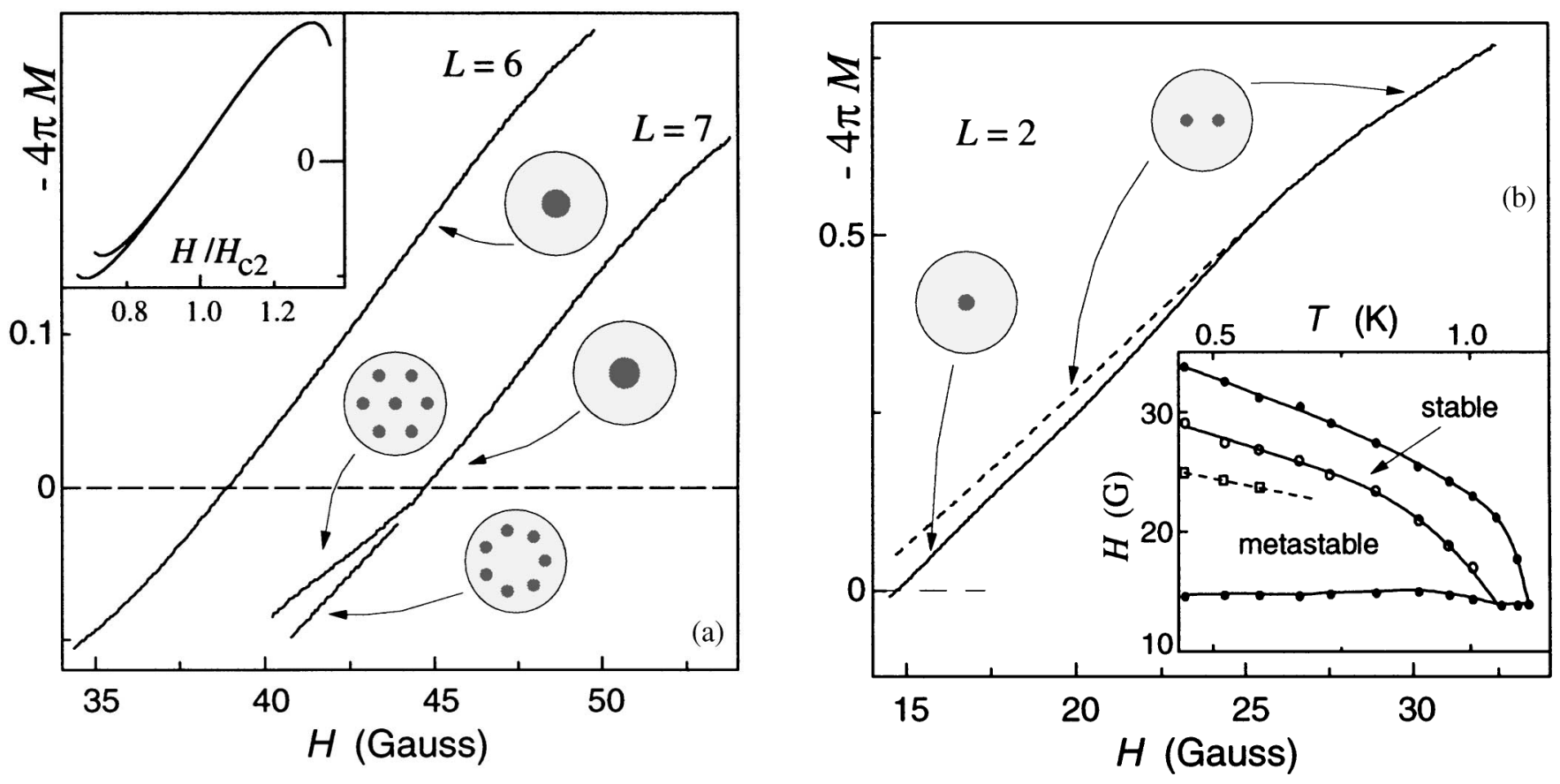

FIG. 2. Transitions in the flux distribution for seven (a) and two (b) fluxoids for the disk in Fig. 1(b). The drawings schematically show the expected distribution of magnetic flux for different branches of the curves. The inset in panel (a) plots a similar transition from a giant vortex to two different multivortex configurations as found in theory [11]. The inset in panel (b) shows the superconducting phase diagram for the $L=2$ state. Solid circles define the range of its existence. Open circles mark the border between thermodynamically stable and metastable situations [this border is defined according to [2] as the disappearance of the preceding $(L=1)$ state]. The dual state where the branching is found lies below the dashed line. All the curves in the inset are guides to the eye.

at $0.3 \mathrm{~K}$. On the other hand, this substate is reached every time when sweeping the field down either from $L=3$ or along the main fluxoid curve. The absence of any noticeable anomaly at the transition between the solid and dotted curves in Fig. 2(b) suggests that this substate is the continuation of the fluxoid configuration at the diamagnetic end. For completeness, Fig. 2(b) presents the superconducting phase diagram for the $L=2$ state. This is the first measurement of the phase diagram for an individual fluxoid state. Other states exhibit somewhat similar diagrams but the complete diagram for a FFS becomes complex because of the coexistence of many different states.

The extra features on the magnetization curves unambiguously indicate structural transitions in the flux distribution for a given $L$. In order to understand the origin of the observed kinks, it is convenient to recall the case of macroscopic superconductors, where at $H_{\mathrm{c} 2}$ a secondorder transition from the surface to bulk superconductivity occurs. At this transition (never observed experimentally), the flux enclosed inside a surface superconducting sheath splits into vortices. A mesoscopic analog of the $H_{\mathrm{c} 2}$ transition would be the partition of a giant vortex with orbital momentum $L$ into $L$ Abrikosov vortices, so-called giantmultivortex transition (GMT) intensively discussed in literature $[3,8,10-12]$. GMTs are expected to appear as kinks on magnetization curves and follow a behavior similar to the one we observed in the experiment. In fact, when during a structural transition a magnetization curve becomes steeper, it means that more flux $(\langle B\rangle=H+4 \pi M)$ is enclosed inside a superconductor, compared to the situation in which the curve would continue straight [inset in Fig. 1(b)]. "More flux" means that individual fluxoids inside the disk are located farther away from the edge because, in such a case, a smaller amount of flux "leeks" outside the disk. Therefore, the direction of all the kinks (except for $L=2$ ) shows that, with increasing $H$, individual vortices merge into a giant vortex and not vice versa, in agreement with theory. Furthermore, we observe no kinks either for $L=0$ and 1 (no vortices to merge) or above $H_{\mathrm{c} 2}$ (giant-vortex regime). In Fig. 1, only the very last GMTs (for $L=5$ and 10) occur in the thermodynamically stable situation. Therefore, these transitions represent the equilibrium (true) $H_{\mathrm{c} 2}$ transition. Both kinks occur close to the value of $H_{\mathrm{c} 2}(0.5 \mathrm{~K}) \cong 60 \mathrm{G}$ for the disks' material. All other GMTs occur in the metastable regime. Although there is general agreement between the experiment and theory [2-4,10-12], the latter finds less pronounced kinks and they always occur close to $H_{\mathrm{c} 2}$.

Concerning the observed branching, note first that the substates always occur in the multivortex regime where one might expect various possible configurations of the same number of vortices $[11,12,14]$. Furthermore, the substates have shallower slopes than the giant-vortex curves [Fig. 1(b)], which implies that fluxoids comprising substates are more loosely distributed inside the disk compared to the case of the giant vortex. This proves that 
the substates are also multivortex arrays, similar to those revealed by the corresponding kinks. This is further supported by the fact that above $H_{\mathrm{c} 2}$, where the disks are no longer in the multivortex regime, no such splitting is observed. The theory confirms the possibility of such multiple vortex geometries [see Fig. 2(a)].

The fine structure for $L=2$ can also be attributed to the merger of two vortices into a double-flux vortex. However, the opposite direction of this kink implies that the equilibrium configuration consists of two individual vortices, while the robust metastable configuration at low fields is a giant vortex [Fig. 2(b)]. A transition from a giant vortex to individual vortices with increasing rather than decreasing field is unexpected. Nevertheless, there is little room for alternative explanations as other fluxoid configurations are not possible for $L=2$. The discussed behavior is observed in three samples, which makes the feature unlikely to be defect related.

It is instructive to estimate the energy scales involved in the fluxoid transitions. By integrating along magnetization curves and, assuming [2-4] that their diamagnetic ends mark the thermodynamic transition, we can find the energy separation between various states. This separation changes with field: It is zero at the diamagnetic ends and maximal at the low-field ends, where our disks persist in metastable configurations with the energy up to $0.5 \mathrm{eV}$ above the equilibrium (for the given film thickness). This represents $\approx 10 \%$ of their condensation energy. On the other hand, the energy gained from switching between various vortex configurations with the same $L$ does not exceed $\approx 10 \mathrm{meV}$.

Finally, we want to point out a rather counterintuitive feature of the fluxoid transitions. Notice that, for example, the transition between the two giant vortex states in Fig. 2(a) $(L=6$ and $L=7)$ can occur only via a firstorder transition while the splitting of a giant vortex $(L=$ 7 ) into 7 vortices can occur as a second-order transition. The corresponding distributions of the density of Cooper pairs (Fig. 3) show the obvious change in symmetry for the second-order transition while any change is hardly noticeable for the first-order transition. This seemingly contradicts the theory of phase transitions, which expects first-order transitions to exhibit more pronounced changes in symmetry than second-order transitions. This puzzle is resolved in Fig. 3 by plotting the imaginary part of $\psi$ (it can equally be its real part or the phase). It is clearly seen now that in the first-order transition there is a change from sixfold to sevenfold symmetry for the complex order parameter as a whole. On the other hand, the "complex" symmetry remains sevenfold along the whole fluxoid curve $L=7$. This illustrates that not only the "real" distribution of Cooper pairs but also the complex (or quantum) symmetry are important in fluxoid transitions.

In conclusion, we have shown that a fixed number of fluxoids inside a mesoscopic superconductor can be found

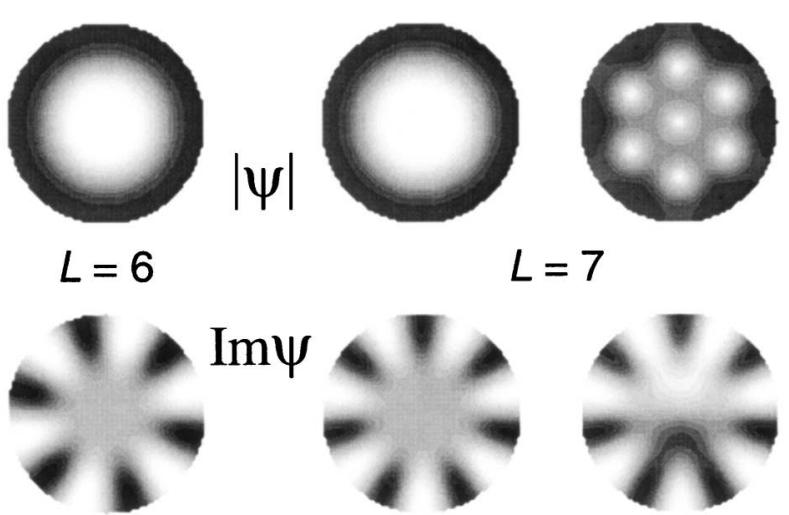

FIG. 3. Calculated distribution of the density of Cooper pairs (top row) and the imaginary part of the order parameter (bottom row) for six (first column) and seven fluxoids. The second and third columns correspond to giant and multivortex states for $L=7$, respectively. The white color represents minima.

in a number of different configurations and not only one, preferential configuration as previously assumed. There is good agreement between the experiment and the theory developed recently for our disks but some observations still have to be understood (e.g., the case of $L=2$ ). To this end, we hope that visualization techniques can be employed to study confined vortex structures, which will clarify the situation.

We acknowledge discussions with F. Peeters, V. Schweigert, and V. Moshchalkov.

[1] A. K. Geim et al., Nature (London) 390, 259 (1997); 396, 144 (1998).

[2] P. S. Deo et al., Phys. Rev. Lett. 79, 4653 (1997).

[3] V. A. Schweigert, F. M. Peeters, and P. S. Deo, Phys. Rev. Lett. 81, 2783 (1998).

[4] J. J. Palacios, Phys. Rev. B 58, R5948 (1998); E. Akkermans and K. Mallick, J. Phys. A 32, 7133 (1999).

[5] R. D. Parks and W. A. Little, Phys. Rev. A 133, 97 (1964).

[6] V. V. Moshchalkov et al., Nature (London) 373, 319 (1995).

[7] O. Buisson et al., Phys. Lett. A 150, 36 (1990); X. Zhang and J. C. Price, Phys. Rev. B 55, 3128 (1997); H. J. Fink, Phys. Rev. 151, 219 (1966).

[8] A. Bezryadin, A. Buzdin, and B. Pannetier, Phys. Rev. B 51, 3718 (1995); H. T. Jadallah et al., Phys. Rev. Lett. 82, 2935 (1999).

[9] A. K. Geim et al., Appl. Phys. Lett. 71, 2379 (1997); F. M. Peeters and X. Q. Li, Appl. Phys. Lett. 72, 572 (1998).

[10] V. A. Schweigert and F. M. Peeters, Phys. Rev. Lett. 83, 2409 (1999); Phys. Rev. B 57, 13817 (1998).

[11] J. J. Palacios, Phys. Rev. Lett. 84, 1796 (2000).

[12] V. A. Schweigert and F. M. Peeters, cond-mat/9910110.

[13] R. P. Huebener, Magnetic Flux Structures in Superconductors (Springer-Verlag, Berlin, 1979).

[14] A. I. Buzdin and J. P. Brison, Phys. Lett. A 196, 267 (1994). 